\title{
Exome sequencing as a diagnostic tool to identify a causal mutation in genetically highly heterogeneous limb-girdle muscular dystrophy
}

\author{
Journal of Human Genetics (2013) 58, 564-565; doi:10.1038/jhg.2013.33; published online 16 May 2013
}

Limb-girdle muscular dystrophy (LGMD) is genetically one of the most heterogeneous diseases, with $>20$ genes identified. ${ }^{1}$ Moreover, causative mutations remain undetermined in more than half of all LGMD patients, suggesting additional heterogeneity. In Japan for instance, $\sim 60 \%$ of the genetic causes of LGMD remain unknown (YK Hayashi et al., unpublished data). LGMD involves recessive as well as dominant disease alleles on autosomes and the $\mathrm{X}$ chromosome, ${ }^{2}$ and the mutations are widely distributed across the entire length of multi-exon genes. The observed mutation frequency for particular LGMD genes varies among different races or geographic regions. The lack of clinical or laboratory findings that help identify the disease-causing genes and their respective common mutations also hinders efficient gene-screening. It is costly and time-consuming to screen all known disease-causing genes by conventional PCR and Sanger sequencing.

Mutation detection is necessary for definitive diagnosis, specifically for genetic counseling, prenatal diagnosis or any-genebased therapy, as well as understanding disease pathogenesis and epidemiology. Recent developments in high-throughput sequence capture methods and nextgeneration sequencing technologies allow for targeted enrichment and resequencing of nearly all exons of protein-coding genes, and have made exome sequencing not only feasible but also extremely cost-effective. Exome sequencing is increasingly being adopted as a diagnostic tool for genetic disease characterized by extreme genetic heterogeneity, as well as for discovery of new causal mutations for unidentified inherited disorders. ${ }^{3}$
We studied a 37-year-old Japanese female patient, who was born to consanguineous asymptomatic parents (first cousins) and developed difficulty in climbing stairs at the age of 20. She had no siblings. She was seen in our department at the age of 23 and was found to have slowly progressive proximaldominant muscle atrophy and weakness, hypertrophy of the calf muscles and high serum creatinine kinase levels (1164 IU/l). She was clinically diagnosed as probable autosomal recessive LGMD (LGMD2).

The study received approval from the ethics committees of Okayama University and the National Center of Neurology and Psychiatry. We obtained written informed consent from all subjects participating in the study. Genomic DNAs were extracted from peripheral blood samples obtained from the patient and her parents. We used exome sequencing (Otogenetics Corp., Norcross, GA, USA) to identify the causative mutation in this patient. We captured the genomic regions comprising all exons and exon-intron boundaries, $5^{\prime}$ and $3^{\prime}$ untranslated regions, $\sim 650 \mathrm{bp}$ of upstream promoter regions, small nuclear RNA and microRNA loci, and conserved intergenic regions using a NimbleGen SeqCap EZ Human Exome Library v2.0 (Roche NimbleGen, Madison, WI, USA). We sequenced the library with 90-bp paired-end reads on a next-generation HiSeq2000 sequencer (Illumina, San Diego, CA, USA) according to the manufacturer's instructions. We obtained 67748784 sequencing reads, comprising 6.1 billion bases. We aligned the sequences against the reference human genome (University of California Santa Cruz hg 19), and scored variants using the DNAnexus genome browser, April 2011
(DNAnexus Inc., Mountain View, CA, USA; http://www.dnanexus.com), using the 'two reads supporting the variation' option. Reads were filtered to remove those with Phred scores of six or less, primer contamination, or poly-A/C/G/T tracts. Reads mapped to ribosomal RNA loci were also excluded from further analysis. After these filtering steps, we obtained 59694348 (88.1\%) mapped reads with an average sequence depth of $\times 56$. After removing variants registered in the public database (dbSNP132), 13316 variants remained (13 120 non-synonymous substitutions and 196 frame-shifts). As the consanguineous family history argued against screening for autosomal dominant or X-linked LGMD, we focused on homozygous mutations in the 16 known genes (LGMD2A-Q) responsible for LGMD2. As a result of genomic variation analysis by the calling algorithm of DNAnexus and manual curation to exclude misaligned reads, only one mutation, a frame-shift c.1795dupA (p.Thr599Asnfs ${ }^{* 33}$, also currently registered as rs80338803 in dbSNP) in exon 15 of the calpain 3 gene (CAPN3), remained as a candidate. We used Sanger sequencing to confirm that this mutation was homozygous in this patient and heterozygous in her parents (Figure 1). Mutations in CAPN3 are responsible for LMGD2A and this same mutation is one of the common mutations in Japanese calpainopathy (LMGD2A), which are rarely found in other races and may reflect a founder effect originating from the relative geographic isolation of Japan. ${ }^{4-6}$

Although several approaches for efficient mutation analysis in patients with LGMD have been employed so far, including immunoanalysis of muscle biopsies ${ }^{7}$ and homozygosity mapping, ${ }^{8}$ protein tests are not always specific and homozygosity 


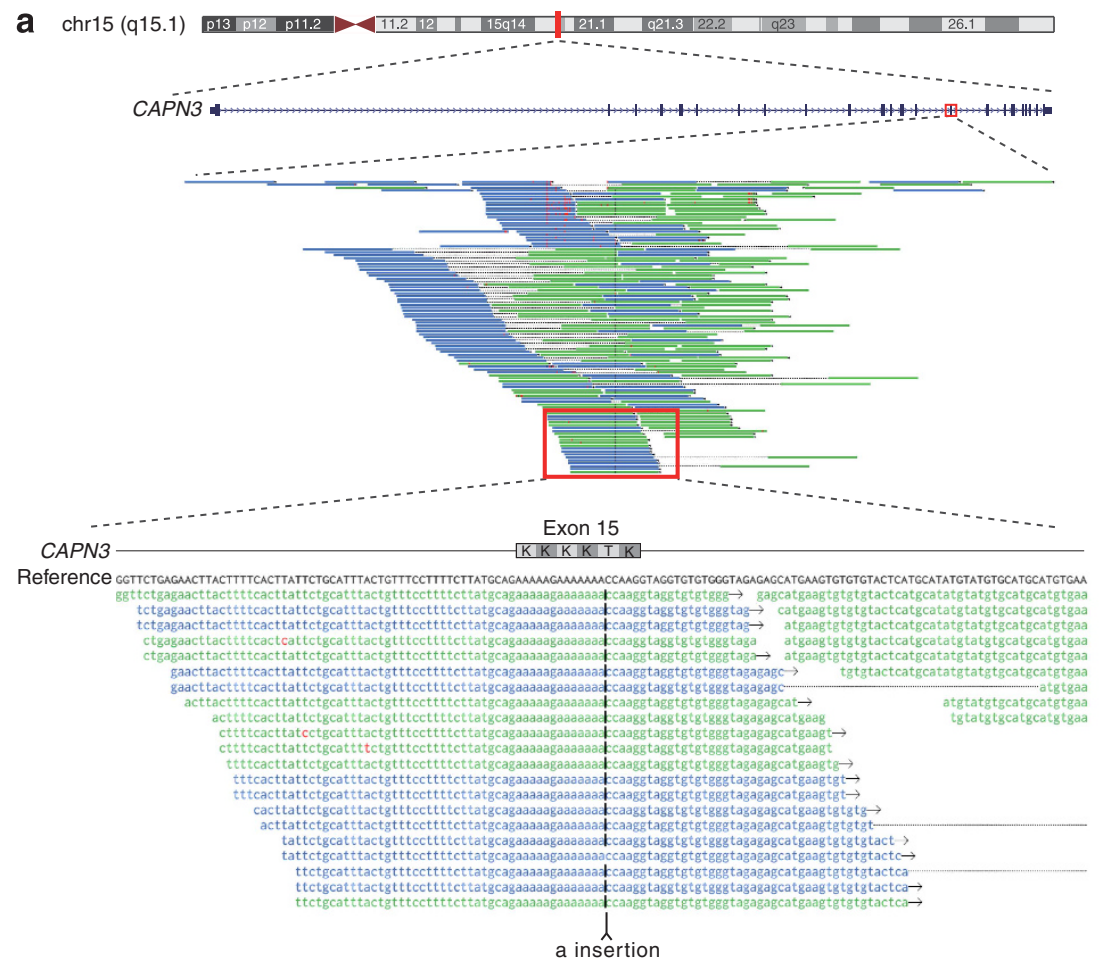

b

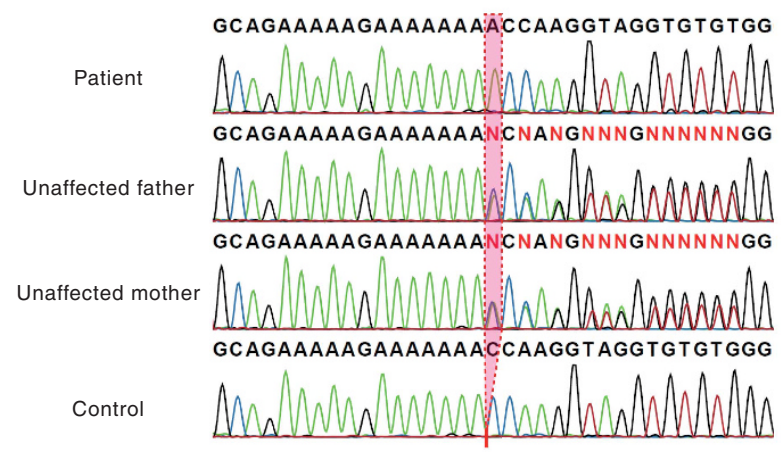

Figure 1 Identification of a homozygous mutation in exon 15 of CAPN3. (a) Next-generation sequencing output at the position of the identified mutation in CAPN3. A coverage depth of 116 sequencing reads covered this particular site, with forward-aligned reads shown in blue and reversealigned reads shown in green. A representative 20 reads out of the 21 aligned in this position (red box) showed the A insertion (black vertical lines), suggesting the homozygous mutation a frameshift c.1795dupA (p.Thr599Asnfs*33). (b) Direct nucleotide sequence analysis confirmed the mutation, which was homozygous in the patient and heterozygous for her asymptomatic parents.

mapping requires the collection of additional family samples and laborious genotyping. Our exome sequencing screen in this patient rapidly identified a previously reported homozygous mutation in CAPN3 and allowed the exclusion of known LGMD genes. The cost of this exome sequencing was $\sim \$ 1000$ for data production and analysis through Otogenetics Corp. and DNAnexus Inc. and thus the method was more costefficient than a series of conventional capillary sequencing of individual multiexon genes, each costing between hundreds

\section{ACKNOWLEDGEMENTS}

We appreciate the cooperation of all patients and doctors who participated in this investigation. This study was supported by Grants-in-Aid for Scientific Research from the Ministry of Education, Culture, Sports, Science and Technology, Japan, as well as Research Grants for Intractable Diseases from the Ministry of Health, Labor, and Welfare, Japan (TM, YKH, IN, KA) and The Okayama Medical Foundation (TM).

Tohru Matsuura ${ }^{1}$, Tatsuaki Kurosaki², Yoshio Omote ${ }^{1}$, Narihiro Minami ${ }^{3}$, Yukiko K Hayashi, ${ }^{3,4}$, Ichizo Nishino ${ }^{3,4}$ and Koji $\mathrm{Abe}^{1}$

${ }^{1}$ Department of Neurology, Okayama University Graduate School of Medicine, Dentistry and Pharmaceutical Sciences, Okayama, Japan; ${ }^{2}$ Department of Biochemistry and Biophysics, University of Rochester Medical Center, Rochester, NY, USA; ${ }^{3}$ Department of Neuromuscular Research, National Institute of Neuroscience, National Center of Neurology and Psychiatry, Tokyo, Japan and ${ }^{4}$ Department of Clinical Development, Translational Medical Center, National Center of Neurology and Psychiatry, Tokyo, Japan E-mail: tohrum@cc.okayama-u.ac.jp

1 Pegoraro, E. \& Hoffman, E. P. Limb-girdle muscular dystrophy overview. in Gene Reviews ${ }^{\mathrm{TM}}$ (eds Pagon, R. A., Bird, T. D., Dolan, C. R., Stephens, K. \& Adam, M. P. http://www.ncbi.nlm.nih.gov/books/ NBK1408/[updated 30 August 2012] University of Washington, Seattle, WA, USA, 1993).

2 Ura, S., Hayashi, Y. K., Goto, K., Astejada, M. N., Murakami, T., Nagato, M. et al. Limb-girdle muscular dystrophy due to emerin gene mutations. Arch. Neurol. 64, 1038-1041 (2007).

3 Ku, C. S., Cooper, D. N., Polychronakos, C., Naidoo, N., Wu, M. \& Soong, R. Exome sequencing: dual role as a discovery and diagnostic tool. Ann. Neurol. 71, 5-14 (2012).

4 Kawai, H., Akaike, M., Kunishige, M., Inui, T., Adachi, K., Kimura, C. et al. Clinical, pathological, and genetic features of limb-girdle muscular dystrophy type $2 \mathrm{~A}$ with new calpain 3 gene mutations in seven patients from three Japanese families. Muscle Nerve 21, 1493-1501 (1998).

5 Minami, N., Nishino, I., Kobayashi, O., Ikezoe, K., Goto, Y. \& Nonaka, I. Mutations of calpain 3 gene in patients with sporadic limb-girdle muscular dystrophy in Japan. J. Neurol. Sci. 171, 31-37 (1999).

6 Chae, J., Minami, N., Jin, Y., Nakagawa, M., Murayama, K., Igarashi, F. et al. Calpain 3 gene mutations: genetic and clinico-pathologic findings in limb-girdle muscular dystrophy. Neuromuscul. Disord. 11, 547-555 (2001)

7 Pogue, R., Anderson, L. V., Pyle, A., Sewry, C., Pollitt, C., Johnson, M. A. et al. Strategy for mutation analysis in the autosomal recessive limb-girdle muscular dystrophies. Neuromuscul. Disord. 11, 80-87 (2001).

8 Boyden, S. E., Salih, M. A., Duncan, A. R., White, A. J., Estrella, E. A., Burgess, S. L. et al. Efficient identification of novel mutations in patients with limb girdle muscular dystrophy. Neurogenetics 11, 449-455 (2010).
CONFLICT OF INTEREST

The authors declare no conflicts of interest. 